\title{
PERFIL SOCIODEMOGRÁFICO DO CÂNCER DE MAMA NA BAHIA NOS ANOS DE 2013 A 2018
}

\section{SOCIODEMOGRAPHIC PROFILE OF BREAST CANCER IN BAHIA IN THE YEARS OF 2013 TO 2018}

\author{
Elisandra Araujo de Assis ${ }^{1}$ \\ Maria da Luz Barreto ${ }^{2}$ \\ Karen Bárbara Eloy Lima ${ }^{3}$
}

\begin{abstract}
O câncer de mama é um tumor maligno que se desenvolve no tecido mamário. Este tipo de câncer é considerado raro em mulheres jovens com idade inferior a 40 anos, porém nessa população a doença cursa geralmente com pior prognóstico. Estudos apontam alta incidência de vítimas no Brasil, apresentando destaque também na saúde pública mundial. O objetivo do presente estudo é analisar o perfil sociodemográfico do câncer de mama na Bahia nos anos de 2013 a 2018. Trata-se de um estudo epidemiológico descritivo, apresentando dados sobre o câncer de mama em mulheres de 5 a 80 anos ou mais, notificados no Sistema de Informações de Saúde (TABNET) dos Serviços de Morbidade Hospitalar do SUS (SIH/SUS) no CID-10, segundo Região de Saúde (CIR). Foram identificados um total de 22.711 casos de mulheres com câncer de mama no período citado anteriormente, com maior predominância na faixa etária de 50 a 59 anos (6.191 casos), apresentando maior quantidade de casos no ano de 2018 (4.132 casos). A maioria dos dados foram observados em mulheres de raça/cor de pele parda, totalizando 16.071 casos, e a maior incidência registrada é a de neoplasias malignas (20.940 casos, de 2013 a 2018). Com base nos dados obtidos, destaca-se a importância da notificação dos casos de câncer de mama no sistema do SUS, a fim de que verbas sejam disponibilizadas para investimentos em campanhas de prevenção e em exames que detectam o câncer em sua fase inicial.
\end{abstract}

Palavras-chave. Câncer de Mama. Saúde da Mulher. Prevalência.

Breast cancer is a malignant tumor that develops in the breast tissue. This type of cancer is considered rare in women younger than 40 years, but in this population the disease usually presents a worse prognosis. Studies point to a high incidence not only in Brazil, but also worldwide. The objective of the present study is to analyze the sociodemographic profile of breast cancer in the state of Bahia from 2013 to 2018. This article is a descriptive epidemiological study presenting data on breast cancer in women aged 5 to 80 years old or more, in the Health Information System (TABNET) of the Hospital Morbidity Services of SUS (SIH / SUS) in ICD-10, according to the Health Region (CIR). A total of 22,711 cases of women with breast cancer in the period mentioned above were identified, with a greater predominance in the age group from 50 to 59 years (6,191 cases), with a larger number of cases in 2018 (4,132 cases). The majority of the cases were identified in brown skinned women, totalizing 16,071 cases, and the highest recorded incidence was of malignant neoplasms (20,940 cases, from 2013 to 2018). Based on the data obtained, the importance of notification of breast cancer cases in the SUS system is highlighted, so that funds are made available for investments in prevention campaigns and in tests that detect cancer in its initial phase.

Keywords. Breast Cancer. Women's Health. Prevalence.

Recebido: 12/05/2019

Aceito: 15/06/2019

1Biomédica - Faculdades Unidas de Pesquisa, Ciências e Saúde - FAPEC. elyacl2014@gmail.com

${ }^{2}$ Farmacêutica - UESB. Especialista em Citologia Clínica-EBMSP. . luzbarretof@hotmail.com. http://lattes.cnpq.br/8298694742113204

${ }^{3}$ Biomédica - Faculdades Unidas de Pesquisa, Ciências e Saúde - FAPEC. Especialização em Acupuntura - Universalis. karen.eloy@hotmail.com. http://lattes.cnpq.br/9807113681840464 


\section{INTRODUÇÃO}

O câncer caracteriza-se por um conjunto de mais de 100 doenças que se assemelham pela proliferação desordenada de células anormais de crescimento agressivo, com alta capacidade de invasão para outros órgãos (INCA, 2018). Dentre os tipos de câncer existentes, o câncer de mama tem sido a neoplasia mais frequente em incidência no sexo feminino, apresentando destaque na saúde pública mundial, estando entre as principais causas de óbitos no Brasil (INCA, 2014). S S e g u n do o INCA (2018), no Brasil, com exceção do câncer de pele não melanoma, o câncer mamário é o mais frequente em mulheres das regiões Sul, Sudeste, Centro-Oeste e Nordeste, com estimativas de 59.700 casos novos, para cada ano do biênio 2018-2019, apresentando risco estimado de 56,33 casos a cada 100 mil mulheres.

O câncer de mama é um tumor maligno, que se desenvolve no tecido mamário (RODRIGUES; CRUZ; PAIXÃO, 2015). É formado por células anormais de crescimento incontrolável que, por meio da corrente sanguínea e do sistema linfático, se espalham para os tecidos vizinhos e se disseminam para outras regiões do corpo (LIMA; POVOA, 2017). Acomete principalmente mulheres na peri-menopausa (fase que marca o fim da vida reprodutiva da mulher e que antecede a menopausa, por volta dos 50 anos de idade, e é a aproximação da última menstruação causada pela diminuição do hormônio estrogênio) (PINHEIRO et al., 2013).

O câncer de mama é raro em mulheres jovens com idade inferior a 40 anos. Porém, nessa população a doença cursa geralmente com pior prognóstico (PINHEIRO et al., 2013). De acordo com pesquisa realizada por Dutra e colaboradores em 2009, em mulheres jovens as características clínicas, patológicas e imuno-histoquímicas são mais agressivas, o estadiamento é avançado, há maior diâmetro tumoral e maiores chances de desenvolver metástase.

A metástase ocorre em $55,3 \%$ das mulheres jovens, ao passo que nas mulheres idosas a porcentagem é de $39,2 \%$. O índice de mortalidade também é maior em mulheres jovens (38\%), enquanto que nas idosas é de cerca de 33\%. Além disso, o impacto psicológico e social é mais intenso e, geralmente, provoca depressão e má qualidade de vida, características que tornam esta uma doença muito temida pela população feminina (ALMEIDA et al., 2015).

Nesse contexto, fatores que podem contribuir para o diagnóstico avançado em mulheres jovens é a menor busca por exames de rastreamento e a alta densidade mamária na faixa etária mais jovem, que compromete a leitura e interpretação dos resultados mamográficos (PINHEIRO et al., 2013). Além disso, muitos profissionais descartam sinais e sintomas da doença, desconsiderando que mulheres jovens possuem riscos para o desenvolvimento desta (CRIPPA et al., 2003).

É importante observar a presença dos sinais e sintomas que são característicos da doença, para que seja possível um diagnóstico na fase inicial. Como sinais e sintomas, destacam-se: presença de nódulos duros e irregulares, geralmente indolores, edema cutâneo semelhante a uma casca de laranja, hiperemia, dor, alteração no mamilo (descamação ou ulceração e/ou secreção papilar, geralmente transparente, podendo se apresentar com coloração rosa ou avermelhada em caso de presença de eritrócitos) (INCA, 2018).

Existem diversos fatores envolvidos no desenvolvimento da neoplasia, pois se trata de uma doença multifatorial, tais como: hereditariedade, mutações genéticas, nuliparidade, menarca precoce antes dos 12 anos de idade, menopausa tardia após 55 anos, primeira gestação após os 34 anos, obesidade, dieta rica em gordura, sedentarismo, terapia de reposição hormonal por mais de cinco anos e ingestão alcoólica excessiva (BARROS; BARBOSA; GEBRIM, 2001), além da idade, sexo, localização geográfica, tipo de alimentação e o uso de tabaco, que também relacionam-se ao desenvolvimento da doença (HUNTER et al., 2010).

Alguns estudos mostraram que a exposição à radiação ionizante, mesmo em baixas doses, aumenta o risco de desenvolver câncer de mama, particularmente durante a puberdade (INCA, 2009). De acordo com o INCA (2017), o risco referente à radiação ionizante é proporcional à dose e à frequência de exposição. 
Portanto, considera-se este estudo relevante devido ao aumento do número de casos de câncer de mama que têm sido notificados no estado da Bahia. Além disso, é de extrema importância que informações referentes ao câncer de mama sejam difundidas, a fim de prevenir a ocorrência dessa patologia. Assim, definiu-se como objetivo deste estudo analisar o perfil sociodemográfico do câncer de mama na Bahia nos anos de 2013 a 2018.

\section{METODOLOGIA}

Para atender ao objetivo do presente artigo, foi realizado um estudo epidemiológico descritivo, de caráter quantitativo, no estado da Bahia/Brasil. Estudos assim realizados tratam de observar, registrar, analisar e ordenar dados, sem interferir nos mesmos, procurando descobrir a frequência com que o fato ocorre, em sua natureza, bem como descrever as características (PRODANOV; FREITAS, 2013).

No desenvolvimento desta pesquisa de natureza quantitativa, formula-se hipóteses e classifica-se a relação entre as variáveis para garantir a precisão dos resultados, devendo evitar contradições no processo de análise e interpretação dos resultados. Trata-se de uma abordagem empregada em diversos tipos de pesquisas, inclusive nas descritivas para analisar a interação de certas variáveis (PRODANOV; FREITAS, 2013).

Foram coletados dados, no mês de março do ano de 2019, referentes ao câncer de mama em mulheres notificadas no Sistema de Informação de Saúde (TABNET), dos serviços de Morbidade Hospitalar do SUS (SIH/SUS) CID 10, segundo Região de Saúde (CIR), utilizando a faixa etária de cinco a 80 anos e mais, entre os anos 2013 a 2018.

Para proceder à análise, primeiramente os dados foram digitados em planilhas de cálculo dos programas Microsoft Office Excel e Microsoft Office Word, em seguida analisados por meio de estatística descritiva, utilizando-se de cálculos de frequência simples, os quais serão apresentados por meio de tabelas e gráficos.

Para a seleção das características sociodemográficas, foram analisadas as seguintes variáveis: idade, raça/cor da pele (branca, preta, parda, amarela e indígena e sem informações). Foram selecionados 28 municípios do estado da Bahia que notificaram os casos da doença no SIH/SUS, segundo CIR, dentre eles: Alagoinhas, Barreiras, Brumado, Camaçari, Cruz das Almas, Feira de Santana, Guanambi, Ibotirama, Ilhéus, Irecê, Itaberaba, Itabuna, Itapetinga, Jacobina, Jequié, Juazeiro, Paulo Afonso, Porto Seguro, Ribeira do Pombal, Salvador, Santa Maria da Vitória, Santo Antônio de Jesus, Seabra, Senhor do Bonfim, Serrinha, Teixeira de Freitas, Valença e Vitória da Conquista. Alguns municípios do estado da Bahia não notificaram casos da doença.

De acordo com as características clínico-patológicas, buscou-se por neoplasia (maligna ou benigna). Quanto às idades foram categorizadas nas seguintes faixas etárias: 5 a 9 anos, 10 a 14 anos, 15 a 19 anos, 20 a 29 anos, 30 a 39 anos, 40 a 49 anos, 50 a 59 anos, 60 a 69 anos, 70 a 79 anos e 80 anos e mais, observando a faixa etária de maior prevalência e com menor incidência, assim como número de óbitos e tipo de neoplasia mais prevalente.

\section{RESULTADOS E DISCUSSÃO}

Após a realização da pesquisa, foi identificado um total de 22.711 mulheres diagnosticadas com neoplasia mamária na Bahia, entre os anos de 2013 a 2018, notificadas no SIH/SUS no CID-10, segundo a CIR.

O câncer de mama é o tipo mais frequente nas mulheres das Regiões Sul (73,07/100 mil), Sudeste $(69,50 / 100$ mil), Centro-Oeste $(51,96 / 100 \mathrm{mil})$ e Nordeste $(40,36 / 100$ mil). Estudos também apontam 
que, no ano de 2030, ocorrerão 21,4 milhões de casos novos e 13,2 milhões de mortes por câncer em todo o mundo, sendo isto em decorrência do crescimento e do envelhecimento da população (INCA, 2014).

Nesta perspectiva, o câncer de mama torna-se um grave problema de saúde pública. Estatísticas indicam um aumento da ocorrência a nível mundial, tanto para países desenvolvidos quanto os países em desenvolvimento, inclusive vêm se apresentando também em faixas etárias mais jovens (MENDES et al., 2014).

De acordo com os dados coletados, apontou-se um aumento de vítimas da doença nos últimos anos, apresentando maior prevalência nos anos de 2018, com 18,1\% (4.132 casos), seguido do ano de 2016 com 17,3\% (3.934 casos) (Gráfico 1).

Gráfico 1. Distribuição do número de casos de câncer de mama nos anos de 2013 a 2018.

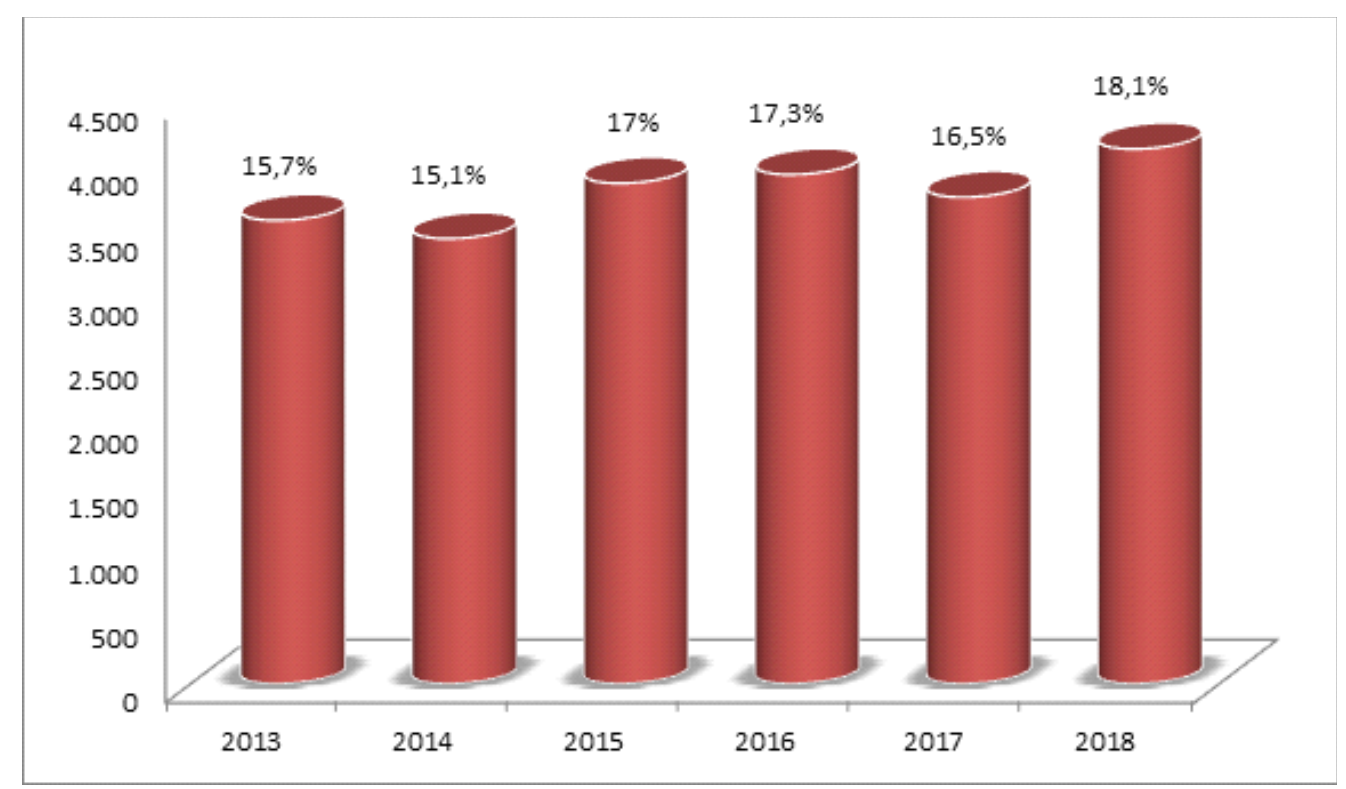

Fonte: DATASUS, 2019.

No que diz respeito à faixa etária, a maior predominância encontrada foi na faixa etária de 50 a 59 anos, totalizando 27,1\% (6.191 casos) (Gráfico 2). Este dado se explica pelo fato de que o câncer de mama acomete principalmente mulheres na perimenopausa (PINHEIRO et al., 2013).

Em segundo lugar em predominância, observou-se a faixa etária de 40 a 49 anos, representando $26,1 \%$ (5.949 casos). Segundo Narod (2012) e Pinheiro et al. (2013), apesar de ser uma doença comum em mulheres na pós-menopausa, há relatos de aumento significativo em mulheres jovens (antes dos 40 anos idade) (Gráfico 2).

Dos 30 aos 39 anos de idade a incidência foi de 11,8\% (2.696 casos). Segundo o Ministério da Saúde (2002), o câncer de mama é uma patologia considerada rara antes dos 35 anos, havendo aumento rápido e progressivo com a idade. Sabe-se que a doença acomete principalmente mulheres entre 40 e 60 anos (BRASIL, 2008). Porém, Segundo Sclowitz et al. (2005), existem referências de que a doença vem atingindo um maior número de mulheres jovens. A idade, portanto, é considerada um dos principais fatores de risco, uma vez que em mulheres jovens, com idade inferior a 25 anos, o câncer mamário é uma doença rara. Por outro lado, torna-se crescente em mulheres mais velhas e, depois, o número de casos decresce novamente (ROBBINS; COTRAN, 2010).

Apesar de raros, foram notificados 50 casos (0,2\%) de mulheres ainda muito jovens, dos 10 aos 14 anos de idade, sendo considerado um dado significativo. Outro dado importante foi um caso de 5 a 9 anos no município de Vitória da Conquista, no ano 2017. 
Gráfico 2. Distribuição dos casos de câncer de mama de acordo com a faixa etária nos anos de 2013 a 2018 na Bahia

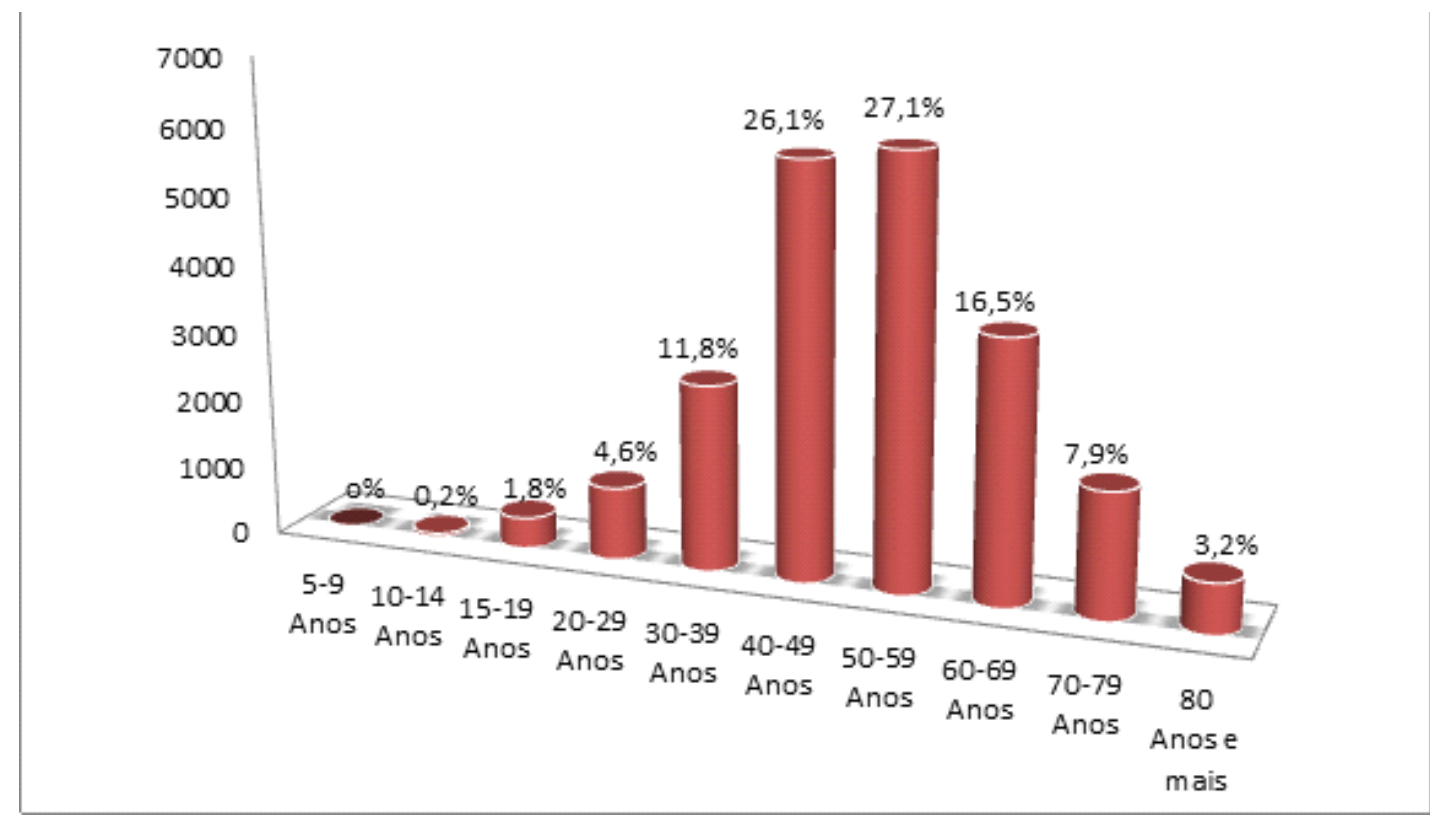

Fonte: DATASUS, 2019.

Quanto à variável raça/cor de pele, foram selecionadas branca, preta, amarela, parda e indígena. Nesta pesquisa observou-se que, quanto à raça indígena, houve apenas um caso notificado, sendo maior o número de casos de câncer de mama em mulheres de cor de pele parda $70,7 \%$ (16.071 mulheres) (Gráfico 3).

Dado semelhante foi identificado em um estudo realizado no Hospital de Santa Rita de Cássia, em Vitória (ES), de 2000 a 2006, no setor de Registro Hospitalar de Câncer do município. No citado estudo, em uma análise composta por 2930 cadastros de mulheres com tumor primário de mama, 53\% correspondiam à pacientes de cor parda e 43,3\% com cor de pele branca (SILVA, 2009).

Gráfico 3. Distribuição dos casos de câncer de mama de acordo com a raça/cor de pele nos anos de 2013 a 2018 na Bahia

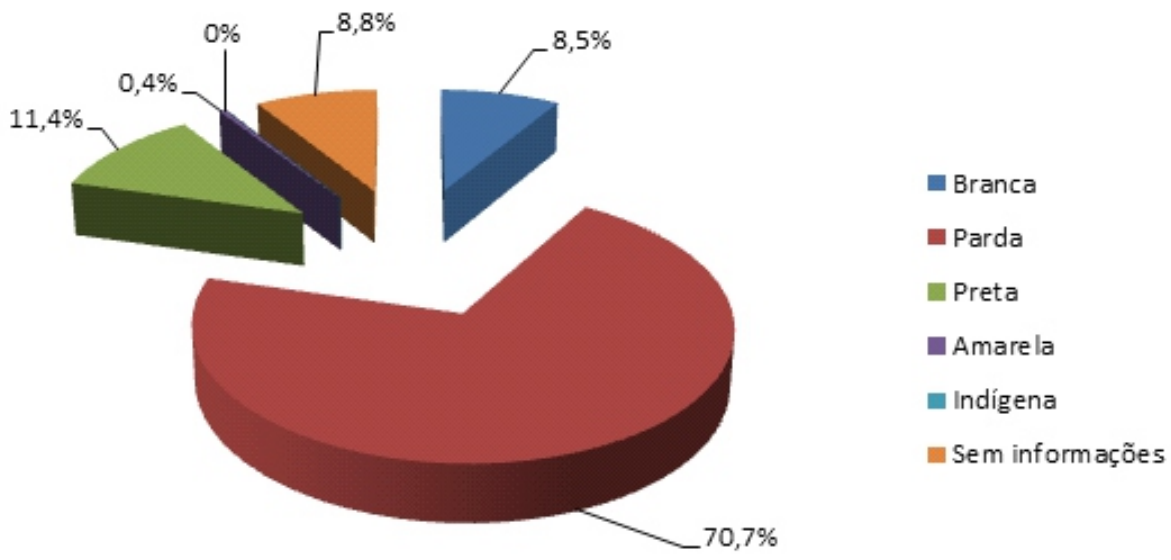

Fonte: DATASUS, 2019. 
Considerando as características clínicas das vítimas, observou-se a quantidade de acometimentos tanto de neoplasia benigna como maligna, apresentando predominância de neoplasia maligna em $92,2 \%$ (20.940) dos casos. Dentre estes, observou-se maior incidência na faixa etária de 50 a 59 anos, correspondendo a 26,5\% (6.020 casos), seguido dos 40 a 49 anos, com 24,4\% (5.561 casos). Porém, a maior incidência de neoplasia maligna foi identificada no ano de 2018 , representando $17,2 \%$ (3.925 casos) (Gráfico 4).

A neoplasia maligna de mama é o câncer mais incidente entre as mulheres no mundo, e houve um aumento nos últimos anos, principalmente nos países desenvolvidos, sendo que essa ocorrência está atrelada à maior disponibilidade das mulheres a exames de rastreamento, como exames mamográficos (PELED et al., 2008). Segundo o INCA (2012), no Brasil considera-se mais incidente no sexo feminino entre os 40 e 69 anos. Já de acordo com Souza et al. (2013), são encontrados cerca de 49 casos para cada 100.000 mulheres por ano.

Gráfico 4. Relação entre os casos de neoplasias de mama benigna e maligna nos anos de 2013 a 2018 na Bahia.

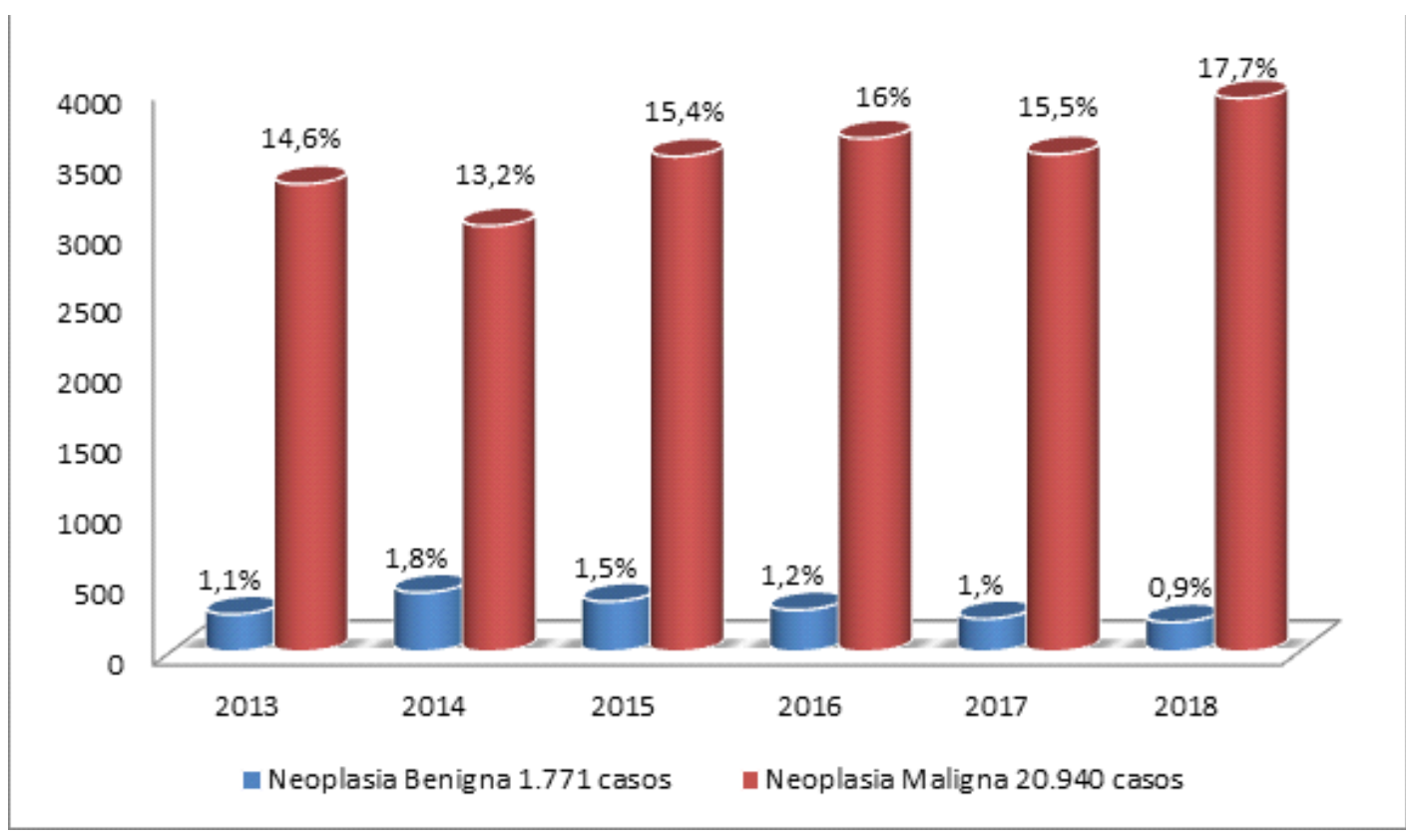

Fonte: DATASUS, 2019.

A análise dos dados apontou um maior predomínio de óbitos na faixa etária dos 40 aos 69 anos de idade, assim como é possível observar que, antes dos 40 e após os 69 anos, ocorreu uma leve redução no número de vítimas da doença em questão (Tabela 1). Sobre isso, o INCA (2018) informa que a incidência do câncer de mama tende a apresentar um crescimento progressivo a partir dos 40 anos, com maior taxa de mortalidade.

Entretanto, antes dos 40 anos de idade, há menor frequência de óbitos, enquanto na faixa etária a partir de 60 anos o risco aumenta. O ano de maior prevalência de óbitos notificados foi o ano de $2017 \mathrm{com}$ $1,3 \%$ (297 casos), seguido do ano de 2015 com 1,2\% (283 casos), que apresentou valores semelhantes ao ano de 2018 , com 1,2\% (280 casos), o que demonstra que ainda há necessidade do cuidado da saúde da mulher (Tabela 1). 
Tabela 1. Distribuição dos casos de óbito por câncer de mama de acordo com a idade nos anos de 2013 a 2018 na Bahia.

\begin{tabular}{|c|c|c|}
\hline Números de óbitos de acordo a idade & $\mathbf{N}^{\circ}$ & $\%$ \\
\hline 5-9 & 1 & $0 \%$ \\
\hline $10-14$ & - & - \\
\hline $15-19$ & - & - \\
\hline $20-29$ & 27 & $0,1 \%$ \\
\hline $30-39$ & 152 & $0,6 \%$ \\
\hline $40-49$ & 358 & $1,5 \%$ \\
\hline $50-59$ & 482 & $2,1 \%$ \\
\hline $60-69$ & 302 & $1,3 \%$ \\
\hline $70-79$ & 181 & $0,7 \%$ \\
\hline A partir de 80 anos & 125 & $0,5 \%$ \\
\hline \multicolumn{3}{|l|}{ Óbitos por ano } \\
\hline 2013 & 258 & $1,1 \%$ \\
\hline 2014 & 249 & $1 \%$ \\
\hline 2015 & 283 & $1,2 \%$ \\
\hline 2016 & 262 & $1,1 \%$ \\
\hline 2017 & 297 & $1,3 \%$ \\
\hline 2018 & 280 & $1,2 \%$ \\
\hline \multicolumn{3}{|l|}{ Óbitos por raça/cor } \\
\hline Parda & 1.077 & $4,7 \%$ \\
\hline Preta & 216 & $0,9 \%$ \\
\hline Branca & 113 & $0,4 \%$ \\
\hline Amarela & 6 & $0 \%$ \\
\hline Indígena & - & - \\
\hline Sem informações & 217 & $0,9 \%$ \\
\hline
\end{tabular}

Fonte: DATASUS, 2019.

Com relação às características demográficas dos pacientes, foi identificado maior número de óbitos em mulheres de cor de pele parda, representando 4,7\% (1.077 casos). Este número de óbitos apresentou maior incidência no ano de 2017 com 0,8\% (198 casos) e no ano de 2018 com 0,8\% (195 casos). Em seguida, observa-se a cor de pele preta 0,9\% (216 casos) com maior incidência no ano de 2015 , com $0,1 \%$ (42 casos). Durante a busca, também foi observado o número de óbitos de acordo com a faixa etária, que apresentou uma curva ascendente, com maior incidência dos 40 aos 69 anos de idade, seguido de uma leve redução dos casos, com maior prevalência no ano 2017 (Tabela 1).

INCA (2011) relata que, nos últimos anos, o câncer de mama tem representado a primeira causa de morte por câncer na população feminina, e que no Brasil houve um aumento crescente nas taxas de mortalidade. Este fato pode ser justificado devido ao atraso no diagnóstico, que atrasa o início do tratamento adequado, promove o crescimento tumoral e torna pior o prognóstico desses pacientes.

INCA (2004) afirma ainda que os países desenvolvidos apresentam uma elevada incidência de câncer de mama, acompanhada por redução nos índices de mortalidade da doença, devido à prática de detecção precoce e à utilização de métodos eficientes para o tratamento.

Durante a busca, foram identificados os nove municípios que apresentaram maior incidência de casos. São estes, em ordem decrescente: Salvador, Feira de Santana, Itabuna, Teixeira de Freitas, Vitoria da Conquista, Ilhéus, Cruz das Almas, Guanambi e Jequié (Gráfico 5). 
Gráfico 5. Municípios com maior incidência de câncer de mama no Estado da Bahia, notificado no sistema de informações de saúde (TABNET), nos anos 2013 a 2018 ( $N=22.711)$.

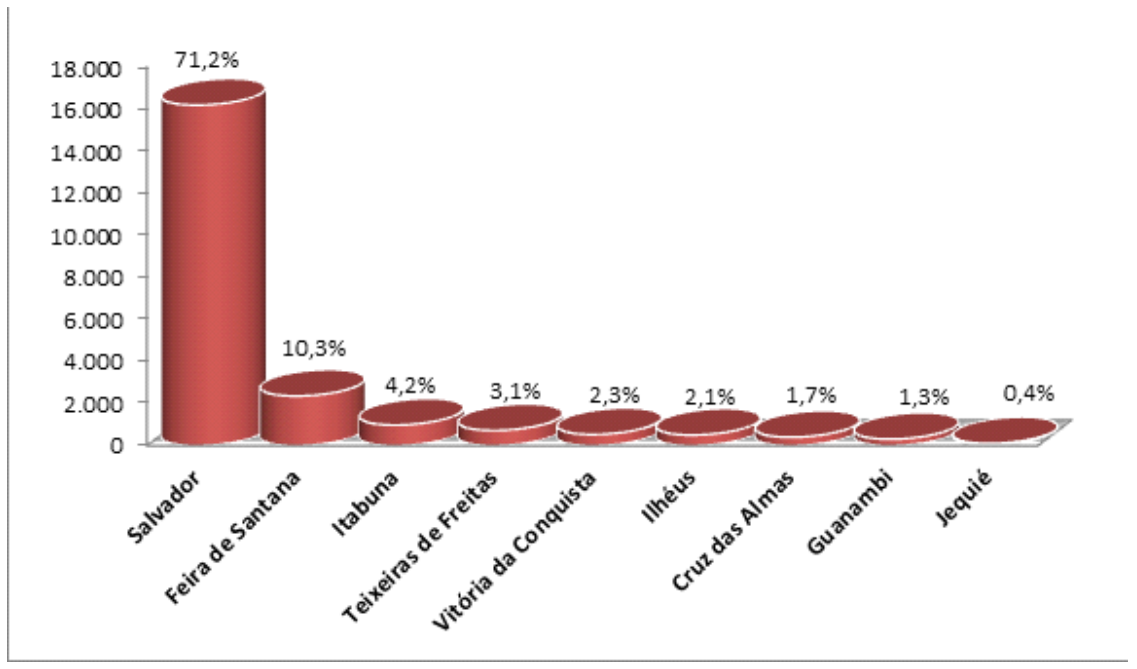

Fonte: DATASUS,2019

O número de casos observados nos municípios que apresentaram maior incidência pode estar relacionado ao local de tratamento, pois os pacientes de municípios de pequeno porte são encaminhados para municípios maiores, por falta de equipamentos e profissionais habilitados para tratar esses pacientes.

Sendo assim, as vítimas podem ser notificadas nos municípios onde são realizados estes tratamentos. Por exemplo, observa-se que Salvador-BA apresenta destaque para todas as categorias de idade e estágios da doença, e esta cidade oferece suporte para quase todos os municípios de pequeno porte circunvizinhos, os mesmos não sendo, provavelmente, notificados nos seus municípios de origem.

\section{CONCLUSÃO}

Diante dos dados apresentados, ressalta-se a importância da notificação dos casos para que verbas possam ser destinadas aos referidos municípios, e para que assim sejam disponibilizados exames preventivos e realização de campanhas educativas para a minimização dos casos e para o cuidado em saúde da população de forma geral.

Após a realização deste estudo, foi observado que o número de casos notificados é baixo diante da incidência mundial, e isso provavelmente se deve à falta de notificação no sistema por parte de muitos municípios do estado.

Vale ressaltar que há municípios onde há relatos de casos, porém estes dados não foram incluidos no sistema, o que faz pensar que a base de dados não foi alimentada de forma adequada, ocorrendo subnotificações e que a quantidade de casos pode ser ainda maior do que os discutidos nesse estudo.

\section{REFERÊNCIAS}

ALMEIDA, T. G. et al. Vivência da mulher jovem com câncer de mama e mastectomizada. Revista de Enfermagem, v. 19, n. 3, jul-set., 2015.

BARROS, A. C. S. D; BARBOSA, E. M.; GEBRIM, L.H. Diagnóstico e tratamento do câncer de mama. São Paulo: Associação Médica Brasileira/Brasília: Conselho Federal de Medicina; 2001: Acesso em: 02 Mar. 2019. 
CRIPPA, C. G. Perfil Clínico e Epidemiológico do Câncer de Mama em Mulheres Jovens. ACM arq catarin med., v. 32, n. 2, p. 50-58, 2003.

DUTRA, M. C. et al. Imunofenótipo e evolução de câncer de mama: comparação entre mulheres muito jovens e mulheres na pós-menopausa. Rev. Bras. Ginecol. Obstet., v. 31, n. 2, p. 54-60, 2009.

HUNTER, D. J. Oral contraceptive use and breast cancer: a prospective study of young women. Cancer Epidemiol Biomarkers Prev., v. 19, n. 10, p. 2496-2502, 2010.

INCA - Instituto Nacional do Câncer. Ações e Programas no Brasil. 2018. Disponível em: <http://www2.inca.gov.br/wps/wcm/connect/acoes_programas/site/home/nobrasil/ programa_controle_cancer_mama>.Acesso em: 18 set. 2018.

INCA - Instituto Nacional do Câncer. Câncer - tipo - mama. 2012. Disponível em: <http://www2.inca.gov.br/wps/wcm/connect/tiposdecancer/site/home/mama>. Acesso em: 03 Mar. 2019.

INCA - Instituto Nacional do Câncer. Incidência de Câncer no Brasil - Estimativa 2014. Disponível em: <http://www.saude.sp.gov.br/resources/ses/perfil/gestor/homepage/outros-destaques/estimativa-de-incidenciade-cancer-2014/estimativa_cancer_24042014.pdf>.

INCA. Instituto Nacional do Câncer. Conceito e Magnitude do câncer de mama. 2018. Disponível em: <https://www.inca.gov.br/controle-do-cancer-de-mama/conceito-e-magnitude>. Acesso em: 28 Fev. 2019.

INCA - Instituto Nacional do Câncer. ProgramadeControledoCâncerdeMama. 2017. Disponível em: < ht t p: / / w w w 2. inca.gov.br/wps/wcm/connect/ acoes_programas/ site/home/nobrasil/programa_controle_cancer_mama>. Acesso em: 18 set. 2018.

INCA. Instituto Nacional do Câncer. Estimativa 2010- Incidência de Câncer no Brasil. 2009. Disponível em: <http://bvsms.saude.gov.br/bvs/publicacoes/estimativa_2010_incidencia_cancer.pdf>. Acesso em: 10 Out. 2018

INCA - INSTITUTO NACIONAL DO CÂNCER. Coordenação de Prevenção e Vigilância. Estimativa 2014: incidência do câncer no Brasil. Rio de Janeiro: INCA, 2014. Disponível em: <http://dx.doi.org/10.1590/s0104$12902017160663>$. Acesso em: 17 Out. 2018.

INCA - Instituto Nacional de Câncer José Alencar Gomes da Silva. Estimativa 2012: incidência de câncer no Brasil [Internet]. Rio de Janeiro: INCA; 2011. INCA; 2011. Disponível em: <http://www.inca.gov.br/ estimativa/2012/estimativa20122111.pdf>. Acesso em: 18 Out. 2018.

INCA - Instituto Nacional do Câncer. Controle do câncer de mama: documento de consenso. Rio de Janeiro: Instituto Nacional de Câncer. 2004. Disponível em: <http://www1.inca.gov.br/publicacoes/Consensointegra.pdf>. Acesso em: 05 Mar. 2019.

LIMA, C.V.C.; POVOA, R. M. F. Mulheres Submetidas à Quimioterapia e suas Funções Cognitivas. Psicologia: Ciência e Profissão, out./dez. 2017. Disponível em: <http://www.scielo.br/pdf/pcp/v37n4/1414-9893-pcp-37-040970.pdf>. Acesso em: 17 Set. 2018.

Ministério da Saúde (BR). Instituto Nacional do Câncer. Ações de enfermagem para o controle do câncer. $3^{\mathrm{a}}$ ed. Rio de Janeiro: INCA, 2008.

Ministério da Saúde (BR). Instituto Nacional do Câncer. Falando sobre câncer de mama. Rio de Janeiro: INCA, 2002.

MENDES, S. I. et al. Correlação da dor e qualidade de vida de mulheres pós-tratamento cirúrgico de câncer de mama. Mundo da Saúde, São Paulo, 2014. Disponível em: <https://www.saocamilosp.br/pdf/mundo_saude/155562/A07.pdf>. Acesso em: 17 Set. 2018.

NAROD, S. A. Breast Cancer in Young women. Nature Reviews Clinecal Oncology, v.9, n.8, p.460-470, 2012.

PELED, R. et al. Breast cancer, psychological distress and life events among young women. BMC Cancer. 2008. Disponível em: <https://www.ncbi.nlm.nih.gov/pubmed/18721454>. Acesso em: 04 Mar. 2019.

PRODANOV, C. C; FREITAS, E.C. Metodologia do trabalho científico: Métodos e Técnicas da Pesquisa e do 
Trabalho Acadêmico. $2^{\mathrm{a}}$ ed. Novo Hamburgo: Feevale, 2013.

PINHEIRO, A B. et al. Câncer de Mama em Mulheres Jovens: Análise de 12.689 Casos. Revista Brasileira de Cancerologia, 2013. Disponível em: <http://www.inca.gov.br/rbc/n_59/v03/pdf/05-artigo-cancer-mama-mulheresjovens-analise-casos.pdf>. Acesso em: 10 Mar. 2019.

RODRIGUES, J. D.; CRUZ, S. M; PAIXÃO, A. N. Uma análise da prevenção do câncer de mama no Brasil. Ciência \& Saúde Coletiva, João Pessoa. 2015. Disponível em: <http://www.scielo.br/pdf/csc/v20n10/1413-8123-csc-2010-3163.pdf >. Acesso em: 07 out. 2018.

ROBBINS; COTRAN. Patologia-Bases Patológicas das Doenças. 8. Ed. Rio de Janeiro: Elsevier, 2010

SOUZA, M. M. et al. Taxa de mortalidade por neoplasia maligna de mama em mulheres residentes da Região Carbonífera Catarinense no período de 1980 a 2009. Cad. Saúde Colet., Rio de Janeiro, v. 21, n. 4, p. 384-390, 2013.

SILVA, P. F. Perfil de mulheres com câncer de mama atendidas em Vitória-ES: influência das variáveis socioeconômicas com o estadiamento clínico do tumor antes do tratamento. 2009. Disponível em: <http://repositorio.ufes.br/jspui/handle/10/5692>. Acesso em 03 Mar. 2019.

SCLOWITZ, M.L.; et al. Condutas na prevenção secundária do câncer de mama e fatores associados. Rev. Saúde Pública, v. 39, n. 3, p. 340-349, 2005. 